\title{
Gestaltungsfelder und Perspektiven für mobiles Lernen in der Hochschule
}

\section{Erscheint in:}

E-Learning in Hochschulen und Bildungszentren. Gestaltungshinweise für pädagogische Innovationen, hrsg. von D.Euler \& S.Seufert.

Version 09.01.2004 - nicht ohne Rücksprache zitieren!

Dr. Christoph Meier

Fraunhofer Institut für Arbeitswirtschaft und Organisation

Nobelstr. 12

70569 Stuttgart

christoph.meier@iao.fhg.de

\section{Einleitung: Vom E-Learning zum M-Learning?}

Mit der Entwicklung von Technologien, Inhalten und Services für ELearning waren zahlreiche (und zum Teil überzogene) Hoffnungen und Erwartungen verbunden. Erwartungen, die sich im universitären Umfeld vor allem auf die Verbesserung der Lehre bezogen und auf mehr Flexibilität bei der zeitlichen Organisation des Lehrens und Lernens. Die rasch fortschreitende Entwicklung im Bereich des ,mobile computing“ und der Netzwerktechnologien liefert die Grundlage für aktuell entwickelte Visionen und Prototypen für Lernen mit mobilen Endgeräten. So werden in Deutschland im Rahmen verschiedener Förderprojekte des Bundesministeriums für Bildung und Forschung erste Schritte in Richtung von sogenannten Notebook-Universitäten genommen. Damit verbunden sind Zielsetzungen wie die Möglichkeit zur Integration privater (mobiler) Rechnerinfrastruktur der Studierenden in die universitäre Ausbildung, das ortsunabhängige und durchgängige Verfügbar-Machen von individuellen Arbeits- und Lernumgebungen, die verbesserte Unterstützung kollaborativen Lernens unter Studierenden oder das ortsunabhängige und durchgängige Verfügbar-Machen von zentralen universitären Dienstleistungen (vgl. Nölting 2002 und http://www.nukath.unikarlsruhe.de/unterseiten/index_en.html, 08.11.2003).

Mobile Lernanwendungen beschränken sich aber nicht mehr auf die Nutzung von Notebook-Computern, sondern umfassen auch die Nutzung 
von kleineren Endgeräten, die in einer Hemd- oder Jackentasche Platz haben. Die Rede ist hier insbesondere von PDAs (Personalen Digitalen Assistenten) und Smartphones (Kombinationen aus PDA und Telefon).

Aufgrund der raschen Entwicklung und Verbreitung solcher „handheld"-Geräte wird verschiedentlich schon von M-Learning als höherer und zeitlich nachfolgender Entwicklungsstufe des E-Learning gesprochen etwa in dem Sinne, dass mit ihnen die bislang noch nicht vollständig eingelöste Vision, wirklich jederzeit an jedem Ort lernen zu können, realisiert werden kann (vgl. Nyiri 2002).

Die Erfahrungen der letzten Jahre haben allerdings gezeigt, dass eine Fokussierung auf das technisch Mögliche im E-Learning häufig nicht zu erfolgreichen Bildungsangeboten führt. Die breite Diskussion über „blended learning" ist ein Resultat dieser Einsicht. Auch ein reines M-Learning wird in der Regel nicht sinnvoll und erfolgreich sein. ${ }^{1}$ Realistisch ist dagegen die Erweiterung von bestehenden Lernarchitekturen und Lernszenarien. Dies gilt insbesondere für das situierte Lernen außerhalb von Kursräumen und Klassenzimmern sowie für informelle Lernaktivitäten am Rande von Lehrgängen und Kursen. Dieser Beitrag will eine Antwort auf die Frage liefern, welche Erweiterungen möglich und sinnvoll sind und in welchen Gestaltungsfeldern Hochschulen (und übrigens auch andere Weiterbildungsanbieter) gefordert sind.

Zunächst werden die ermöglichenden Technologien für mobiles Lernen (Endgeräte, Speichermedien, Netzwerke) kurz vorgestellt. Daran schließt der Hauptteil zu den Gestaltungsfeldern und Herausforderungen beim mobilen Lernen an. Behandelt werden hier Infrastrukturen, Lerninhalte, Lernformen, organisatorische und schließlich auch strategische Aspekte.

\section{Hintergrund: Ermöglichende Technologien für M-Learning}

Lernbezogene Aktivitäten unter Nutzung mobiler Endgeräte (M-Learning) werden durch drei technologische Entwicklungsstränge ermöglicht. Diese betreffen zum einen die Entwicklung von Endgeräten, zum anderen die Entwicklung von Datenträgern und schließlich die Entwicklung von Netzwerk-Technologien.

So wurde beispielsweise im Rahmen des EU-Leonardo-Projekts „mLearning“ (vgl. http://learning.ericsson.net/leonardo/index.html) versucht, komplette Kurse per PDA oder Smartphone zu bearbeiten, wobei sich aber schwerwiegende Usability-Probleme zeigten (vgl. Keegan 2002, Kapitel 9). 


\subsection{Miniaturisierung von Endgeräten und Datenträgern}

Die Entwicklung bei Personal Computern (Prozessoren, Displays, Akkumulatoren) führen zu immer größerer Leistung und ermöglichen gleichzeitig immer geringere Abmessungen der Endgeräte. Besonders rasch schreitet die Entwicklung im Bereich der Mobiltelefone und der Personalen Digitalen Assistenten voran. Waren sie vor wenigen Jahren nur für das Telefonieren oder die Speicherung von Adressdaten und Kalenderinformationen geeignet, so nehmen sie mehr und mehr Züge eines Schweizer Taschenmessers für das digitale Zeitalter an - sie sind alles in einem: Taschenrechner, einfache Digitalkamera, Konsole für Spiele ebenso wie für das Abhören von Musik und das Anschauen von Videos sowie nicht zuletzt auch Basis für die Betrachtung und Bearbeitung von OfficeDokumenten oder für das Aufrufen von Webseiten - letzteres immer häufiger auch über drahtlose Anbindungen an das Internet (via W-LAN oder GSM / GPRS). Gleichzeitig sind heute miniaturisierte Datenträger wie Micro-Festplatten und Flash Speicherbausteine verfügbar, die sehr viel kleiner sind als CD-ROMs, diese bei der Speicherkapazität aber schon übertreffen und fast an DVDs heranreichen. Damit lassen sich auch umfangreiche elektronische Texte, Audio- sowie Videomaterialien überall dort, wo man ein Telefon oder einen PDA dabei haben kann, nutzen.

Mittlerweile haben sich eine ganze Reihe verschiedener Typen von tragbaren Endgeräten entwickelt. Diese reichen von Notebook- und TabletPCs über ,ultraportable“ und am Gürtel tragbare PCs bis hin zu PDAs und Smartphones (vgl. Abbildung 1). 
Abb. 1. Typen mobiler Endgeräte ${ }^{2}$

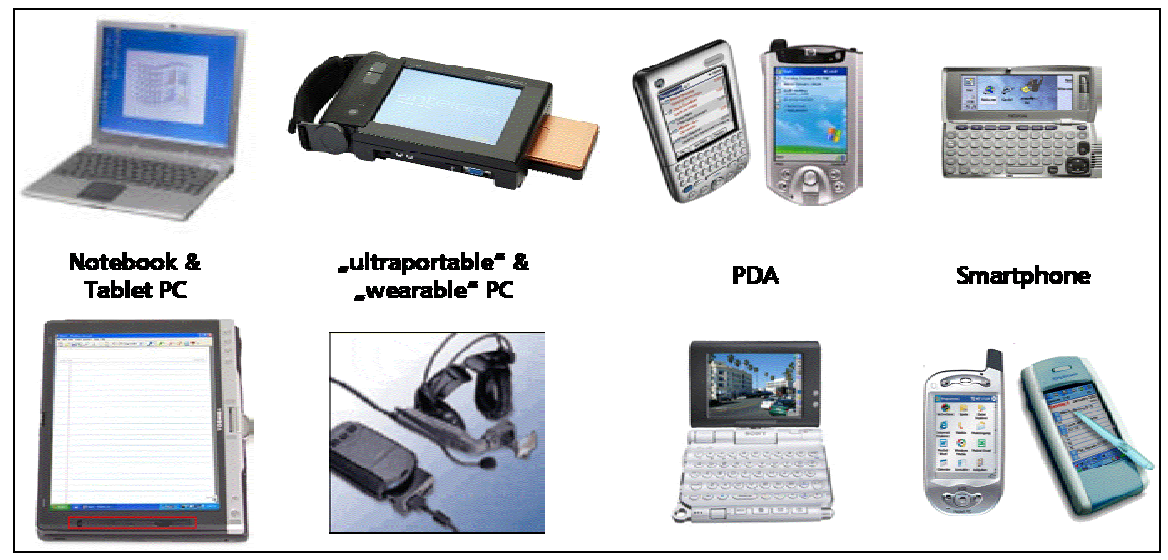

Nicht alle tragbaren Endgeräte - von Notebook-Computern und als Notizblöcken nutzbaren Tablet-PCs über ultraportable PCs, PDAs, Smartphones bis hin zu Mobiltelefonen - sind gleichermaßen für die mobile Nutzung von Lerninhalten und Lernanwendungen geeignet. Während Notebookund ultraportable Computer aufgrund der eingesetzten Betriebssysteme, Anwendungen (z.B. Textverarbeitung oder Web-Browser) und DisplayGrößen ohne Einschränkungen wie herkömmliche Desktop-PCs genutzt werden können, ist dies bei PDAs oder Smartphones nur eingeschränkt der Fall. Insbesondere die kleinen Displays und das (in den meisten Fällen) Fehlen einer integrierten Tastatur zur Dateneingabe sind hier derzeit die beschränkenden Faktoren. Zwar sind für verschiedene handheld-Geräte inzwischen Web-Browser und Viewer-Programme verfügbar, die Webseiten oder Office-Dokumente auf die Größe des verfügbaren Displays verklei-

2 Sogenannte „ultraportable“ oder „,wearable“ PCs verfügen über ein StandardBetriebssystem (z.B. MS-Windows XP) und reichen von ihren Leistungsmerkmalen an Notebooks heran, sind aber auf die Abmessungen eines PDA geschrumpft (z.B. 7,6x12,7x1,9 cm). Typische Leistungsmerkmale aktueller PDAs und Smartphones sind dagegen Prozessoren mit bis zu $400 \mathrm{MHz}$ Taktfrequenz, bis zu $128 \mathrm{MB}$ Arbeitsspeicher und Displays mit Auflösungen bis zu 640x480 Pixel. Tastaturen sind zum Teil in die Geräte integriert, zum Teil werden sie auf dem Display simuliert. Über Steckplätze können zusätzliche Komponenten wie Speicherkarten angeschlossen werden. Während bei aktuellen PDAs häufig schon W-LAN Komponenten integriert sind, können Smartphones in GSM oder GPRS-Netzen betrieben werden. 
nern (,smart screen rendering“). Allerdings sind die Dokumente oder Web-Seiten insbesondere bei Displays mit kleinen Abmessungen nicht wirklich gut zu lesen. Ähnliches gilt für die Erstellung von Dokumenten. Das Erstellen von Text-Dokumenten die umfänglicher sind als eine kurze Email ist mit den auf drucksensiblen Displays simulierten Tastatur kaum möglich und auch mit den mit ein oder zwei Fingern zu bedienenden Kleinst-Tastaturen nicht wirklich praktikabel. Auf den beiden Dimensionen "Tauglichkeit für die Nutzung von Office-Dokumenten und des WWW" sowie "potenzielle Verfügbarkeit auf Grund von Größe und Gewicht" markieren demzufolge Notebooks und Tablet-PCs einerseits und PDAs sowie Mobiltelefone andererseits die jeweiligen Pole.

Abb. 2. Nutzungsmöglichkeiten mobiler Endgeräte

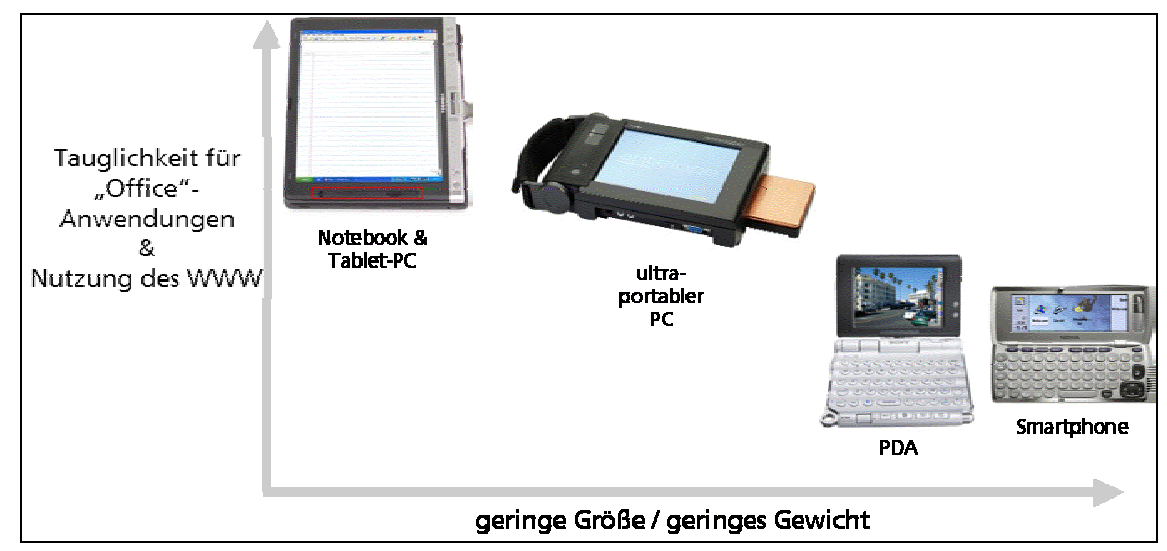

\subsection{Netzwerk-Technologien}

Mobile Endgeräte können auf vielfältige Weise an stationäre ITInfrastrukturen angebunden werden. Neben Verbindungen auf der Grundlage von Infrarot- und Bluetooth-Schnittstellen sind hier insbesondere Wireless-LAN (W-LAN) und paketorientierte Telefonie-Netzwerke zu nennen (vgl. hier und im Folgenden Jung / Warnecke 2002). 
Abb. 3. Anbindung von mobilen Endgeräten

\begin{tabular}{|c|c|c|c|}
\hline $\begin{array}{l}\text { Typen drahtloser } \\
\text { Verbindungen }\end{array}$ & $\begin{array}{c}\text { Art der } \\
\text { Verbindung }\end{array}$ & Bandbreite & $\begin{array}{l}\text { Reichweite / } \\
\text { Abdeckung }\end{array}$ \\
\hline Infrarot (IrDA, FIR) & $\begin{array}{c}\text { gerichtet; } \\
\text { max. } 2 \text { Geräte }\end{array}$ & $0,1-4 \mathrm{Mbit} / \mathrm{s}$ & $2-8 \mathrm{~m}$ \\
\hline Bluetooth & $\begin{array}{l}\text { ungerichtet; } \\
\geq 2 \text { Geräte }\end{array}$ & $1 \mathrm{Mbit} / \mathrm{s}$ & $10-100 \mathrm{~m}$ \\
\hline $\begin{array}{c}\text { W-LAN } \\
(802.11 \mathrm{~b}-\mathrm{g})\end{array}$ & $\begin{array}{l}\text { ungerichtet; } \\
\geq 2 \text { Geräte }\end{array}$ & 11- $54 \mathrm{Mbit} / \mathrm{s}$ & $50-300 \mathrm{~m}$ \\
\hline GSM / GPRS / UMTS & $\begin{array}{l}\text { ungerichtet; } \\
\geq 2 \text { Geräte }\end{array}$ & $\begin{array}{c}\text { ca. } 9 / 40 / 360 \\
\text { Kbit/s }\end{array}$ & $\begin{array}{c}\text { öffentliches } \\
\text { Mobilfunknetz }\end{array}$ \\
\hline
\end{tabular}

Diese Formen der Vernetzung unterscheiden sich vor allem in Bezug auf den Verbindungsaufbau und die verwendeten Protokolle, Reichweiten sowie verfügbare Datenübertragungsraten. Während Infrarotverbindungen beispielsweise immer nur gerichtet und zwischen zwei Endgeräten im Abstand von maximal zwei Metern hergestellt werden können, ist es mit Bluetooth, W-LAN und GSM / GPRS / UMTS-Verbindungen möglich, mehrere Endgeräte in zunehmend größeren Entfernungen anzubinden.

Bluetooth-Verbindungen sind für die Vernetzung von Endgeräten in unmittelbarer Nähe ausgerichtet. Auf diese Weise können beispielsweise von einem Notebook-Computer Dokumente auf einen Drucker übertragen und ausgedruckt werden, ohne dass eine Kabelverbindung hergestellt werden muss.

Drahtlose lokale Netzwerke (W-LAN) sind primär auf Datenübertragung ausgerichtet. Die für Sprachübertragung erforderliche "Quality of Service" ist in der Regel nur mit zusätzlicher Hard- und Software für die Priorisierung von Sprachpaketen gegeben. Aktuell werden Standards entwickelt, die zunehmend höhere Datenraten und größere Reichweiten erlauben.

Das General Packet Radio System (GPRS) erweitert den GSM-Standard um Möglichkeiten der paketorientierten Datenübertragung. Gleichzeitig sind damit Verbindungen möglich, die nicht nach Zeit, sondern nach dem Volumen der übertragenen Daten abgerechnet werden. Je nach Ausbaustufe des Netzes können mit GPRS bis zu 115 kbit/s an Bandbreite zur Verfügung gestellt werden.

Mit der Entwicklung des UMTS-Standard ging diese Entwicklung weiter, wobei hier umfangreiche Neuerungen der Netzarchitektur und der Zugriffsverfahren zugrunde liegen. So hängt beispielsweise die Zellgröße von der Zahl der angemeldeten Teilnehmer und den Bandbreitenanforde- 
rungen ab (lastabhängige Zellatmung). Bei UMTS sind verschiedene Klassen von Diensten definiert, die jeweils unterschiedliche Anforderungen an die Qualität der Verbindung stellen und vermutlich auch unterschiedlich tarifiert werden. Dazu gehören Verbindungen für Sprachübertragung, Verbindungen für Datenübertragung, Verbindungen für Zugriffe auf das WWW und Verbindungen für Email-Empfang.

\section{Mobiles Lernen an der Hochschule: Szenarien, Gestaltungsfelder und Herausforderungen}

Neben den oben angeführten Entwicklungen im Bereich der Endgeräte, der Datenträger und der Netzwerk-Technologien wurden in den letzten Jahren zahlreiche Forschungs- und Entwicklungsprojekte sowie Pilotierungen durchgeführt und das Potenzial für mobiles Lernen erkundet (vgl. z.B. Keegan 2002; Vahey / Crawford 2002; Pelton / Pelton 2003; Trondsen 2003). Die dabei erprobten Anwendungs-Szenarien für Lernen mit Unterstützung durch mobile Endgeräte sind vielfältig und umfassen gleichermaBen das Management von Lernaktivitäten, informelles Lernen und formalisierte Lernaktivitäten. Unterscheidet man diese Szenarien nach den Anforderungen an die Datenein- und -ausgabe, die dabei bewältigt werden müssen, so lässt sich in etwa folgende Reihung erstellen:

- Benachrichtigung von Lernern per SMS oder Email über neue Lernangebote und Anmeldetermine

- Suche nach und Buchung von Lernressourcen in einem Learning Management Systems (LMS) per Mobiltelefon, PDA oder Notebook

- Rezipieren von Lerninhalten wie z.B. WBT oder Aufzeichnungen von Vorlesungen per Smartphone, PDA oder Notebook

- Bearbeiten von kurzen Aufgaben und Lernerfolgskontrollen sowohl im Verlauf einer Lehrveranstaltung

- Messen und audiovisuelles Dokumentieren im Rahmen von Übungen, Praktika oder Exkursionen

- Erstellen und Bearbeiten von Dokumenten wie beispielsweise Seminararbeiten

Die Gestaltungsfelder und Herausforderungen, die mit der Umsetzung dieser und anderer Szenarien mobilen Lernens verbunden sind, liegen in den gleichen Feldern wie beim E-Learning ganz allgemein und betreffen vor allem die Bereiche Strategie, IT-Infrastruktur, Lerninhalte und Lernformen sowie schließlich auch organisatorische Fragen (vgl. Bullinger 2002). 


\subsection{Strategische Aspekte}

Wichtige strategische Fragen, denen sich Hochschulen in den nächsten Jahren stellen müssen, betreffen die eigene Positionierung in einem zunehmend geöffneten Bildungsmarkt und die Verankerung in den jeweiligen politischen Einheiten (z.B. Bundesländer, Kantone), von denen sie getragen werden.

In Bezug auf den zuerst genannten Aspekt sind Hochschulen neben der Verbesserung der Lehre auch damit gefordert, den zunehmenden Bedürfnissen nach Flexibilität auf Seiten der Studierenden Rechnung zu tragen. Die Anmeldung zu einem Seminar, die Benachrichtigung über einen neuen Beitrag im dazugehörigen Diskussionsforum, der Zugriff auf die Literaturliste, das Anschauen der Aufzeichnung eines Gastvortrags, das Abschicken eines Arbeitsblattes - all dies werden Studierende künftig nicht mehr nur vom PC-Pool der Bibliothek oder vom heimischen PC aus nutzen wollen. Vielmehr werden sie Wert darauf legen, dass sie auf diese Ressourcen jederzeit und entsprechend ihren Arbeitsrhythmen zugreifen können: als beispielsweise auf der täglichen Zugfahrt, in Leerlaufzeiten ihres TeilzeitJobs oder im Lesesaal der Bibliothek einer anderen Stadt (d.h., dort, wo der Partner / die Partnerin lebt).

Darüber hinaus sind Hochschulen gefordert, neue Märkte für ihre Dienstleistungen zu erschließen und - insbesondere in flächenmäßig kleinen politischen Einheiten wie Stadtstaaten oder Kantonen - ihre Bedeutung für die Region zu untermauern. Eine intensivere lokale und regionale Vermarktung der eigenen Angebote und Leistungen nicht nur an Alumni, sondern auch an die Menschen in der jeweiligen Region kann ein Mosaikstein in einer diesbezüglich ausgerichteten Strategie sein. So wird es, um nur ein Beispiel zu nennen, mit der Verfügbarkeit von ortsbezogenen Dienstleistungen in den UMTS-Netzen möglich, tagesaktuell Hinweise zu öffentlichen Vorträgen an Bürger zu kommunizieren, die sich gerade in der Nähe der Hochschule befinden und sich für solche Angebote interessieren.

Abb. 4. Beispiel für SMS-basierte Benachrichtigungen zu Lehrveranstaltungen (Quelle: Virtuelle Universität Regensburg)

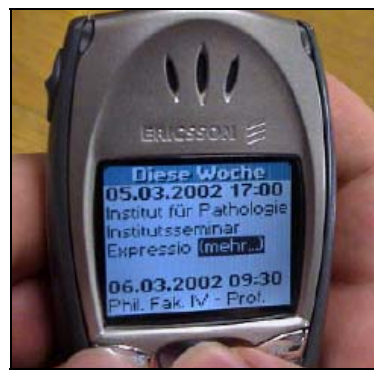




\subsection{Infrastrukturen für mobiles Lernen: Netzwerke, Endgeräte und Lern-Management-Systeme}

Um mehr Flexibilität bei Lehr- und Lernaktivitäten unterstützen zu können, sind Erweiterungen der IT-Infrastrukturen erforderlich. Dies gilt insbesondere für die flächendeckende Einrichtung von drahtlosen lokalen Netzwerken (W-LAN) in Hochschulen. Der Zugriff auf Daten über das öffentliche drahtlose Datennetz (GPRS, UMTS) wird auf absehbare Zeit zu kostenintensiv sein (Schneider 2003, S. 187). Hochschulen, deren Gebäude auf einem Campus konzentriert sind, können eine solche flächendeckende Versorgung mit W-LAN derzeit einfacher und kostengünstiger umsetzen als solche, deren Einrichtungen weiter verstreut sind. Diese Situation kann sich aber mit dem Verfügbar-Werden von „wide area W-LAN“ in den nächsten Jahren verändern.

Die Ausrüstung und Versorgung der Studierenden mit Endgeräten (Notebook-Computer und PDAs bzw. Smartphones) ist ein wichtiger Aspekt der Umsetzung der im Abschnitt 3 genannten Szenarien. Obwohl bereits zahlreiche Studierende über Notebook-Rechner, PDAs und Mobiltelefone verfügen ist die Versorgung doch noch nicht wirklich flächendeckend. Um neue Lehr- und Lernmodelle tatsächlich umsetzen zu können und Benachteiligungen auszuschließen, müssen Hochschulen hier Unterstützung anbieten. Denkbare Modelle sind die Versorgung der Studierenden mit leihweise zur Verfügung gestellten Endgeräten oder Finanzierungsangebote, bei denen die Studierenden über eine monatliche Ratenzahlung die entsprechenden Geräte erwerben.

Das Management des Zugriffs auf administrative Funktionen und Lerninhalte, die auch über mobile Endgeräte wie PDA oder Smartphones genutzt werden sollen, stellt besondere Anforderungen an Learning Management Systeme. Bislang unterstützen nur wenige Lernplattformen den direkten Zugriff mit Endgeräten wie PDA oder Mobiltelefon und das Tracking des Stands der Bearbeitung von Lerninhalten.

\subsection{Lerninhalte}

Während die in den letzten Jahren im Rahmen zahlloser Projekte und auch von kommerziellen Anbietern entwickelten digitalen Lerninhalte ohne Einschränkungen auch mit Notebook-, Tablet- und ultraportablen Computern genutzt werden können, gilt dies für Endgeräte wie PDAs und Smartphones nur mit Einschränkungen. So lange die Auflösungen der Displays der mobilen Endgeräte und die eingesetzten Viewer-Technologien eine gut lesbare Darstellung von Standard-Dokumenten und CBT / WBT noch nicht erlauben, sind zwei Wege denkbar, um bereits existierende 
Lernangebote auch mobil nutzbar zu machen. Im einen Fall wird eine Reformatierung der Inhalte erforderlich, was beispielsweise die Auflösung komplexer Inhalte-Seiten in einzelne Objekte wie Text, Grafik etc. erfordern kann. Einen anderen Weg stellt die Filterung von Inhaltsobjekten dar, wobei dann nur diejenigen Objekte ausgegeben werden, die dem aktuellen Endgeräte-Profil entsprechen (vgl. Lehner et al. 2003 und Bomsdorf 2003). Auch das Verfolgen von Vorlesungen, die per Media-Streaming übertragen werden, mit PDAs oder Smartphones (vgl. Lehner et. al. 2003 und die Angebote der Virtuellen Universität Regensburg unter http://vur.uni-regensburg.de), wird aufgrund der damit verbundenen Kosten vermutlich nur in wenigen Situationen sinnvoll sein. ${ }^{3}$

Abb. 5. Media-Streaming einer Vorlesung auf ein GPRS-Telefon (Quelle: Lehner et al. 2003)

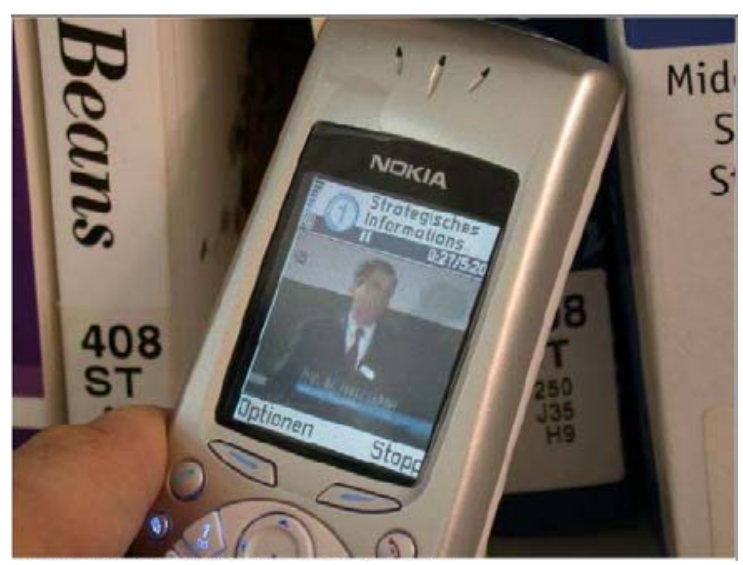

Für das Lernen mit jederzeit verfügbaren Endgeräten wie PDAs und Smartphones erscheinen Angebote aussichtsreich, die wenig Einarbeitung erfordern, schnell begonnen und jederzeit unterbrochen werden können. Ein elektronisches Lernkarteikarten-Systeme erlaubt beispielsweise das Erstellen von digitalen Karteikarten zu beliebigen Inhalten, die dann mit

3 Zwar ist es durchaus möglich, Aufzeichnungen kompletter Vorlesungen auf Speicherbausteinen abzulegen und damit Übertragungskosten zu sparen. Aber die Displays von PDAs und Smartphones sind gegenwärtig noch zu klein, um eine didaktisch sinnvolle Kombination verschiedener Informationstypen wie etwa Folienpräsentation, Videobild und Inhaltsverzeichnis abzubilden (vgl. Meier et al. 2003). 
einem PDA durchgearbeitet werden können. Dabei werden Karteikarten mit Fragen, die richtig beantwortet wurden, weiter zurück gestellt, während Karteikarten mit Fragen, die nicht richtig beantwortet wurden, häufiger eingeblendet werden.

Abb. 6. Lernkarteikarten-System für PDA (Quelle: Bomsdorf 2003)

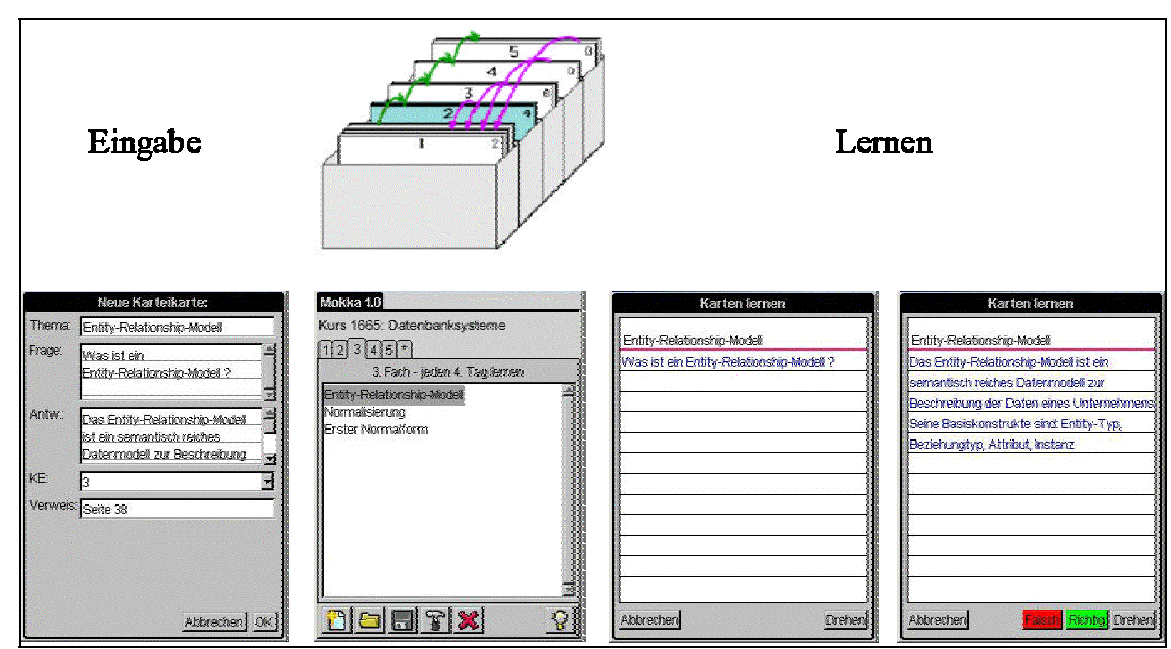

Lernmodule oder „learning bytes“, die innerhalb weniger Minuten durchgearbeitet werden können, eignen sich ebenfalls für die Nutzung in kurzen Leerlaufzeiten, also beispielsweise in der Straßenbahn oder im Zug. Ein Beispiel ist ein illustriertes Lernmodul zum Thema Therapiemöglichkeiten bei Osteoporose der Firma Centrax, das innerhalb von ca. 10 Minuten durchgelesen werden kann. 
Abb. 7. Screenshot eines kurzen Lernmoduls zur Therapie von Osteoporose für PDAs (Quelle: http://www.centrax.com)

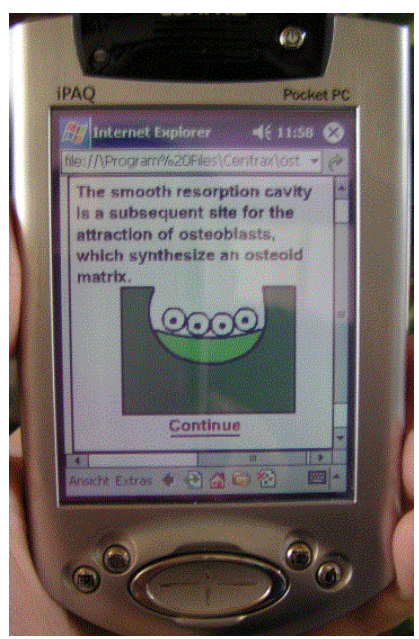

Bislang gibt es allerdings nur wenige Autorenwerkzeuge für das Erstellen von Lerninhalten, die für Geräte mit kleinen Bildschirmen optimiert sind (z.B. http://www.lectora.com). Viel wichtiger aber ist, dass es bislang keine ausgearbeiteten didaktische Konzepte für Lerninhalte gibt, die primär mit Endgeräten wie PDA oder Mobiltelefon genutzt werden sollen. Hier gibt es noch erheblichen Entwicklungsbedarf. ${ }^{4}$

\subsection{Lernformen}

Mit dem Einsatz mobiler Endgeräte wie (Notebook-Computer) PDAs und Smartphones ergeben sich nicht nur Möglichkeiten zur Nutzung von kurzen Leerzeiten für Lernzwecke, sondern auch Möglichkeiten zur Weiterentwicklung und Transformation etablierter Lernformen wie etwa Seminare, Vorlesungen oder Exkursionen. So können etwa Lehrende in Form von kurzen Arbeitsaufgaben oder Tests Rückmeldungen von den Lernenden einfordern und so das Verständnis gerade behandelter Inhalte und den Be-

4 So sollen beispielsweise im Rahmen eines vom Bundesministerium für Wirtschaft und Arbeit geförderten Projekts (,Qualitätsinitiative E-Learning Deutschland") didaktisch fundierte Ausspielungsregeln entwickelt werden, die eine Transformation bestehender html-basierter Lerninhalte zur Nutzung via PDA / Smartphone ermöglichen. 
darf nach Vertiefungen überprüfen. Genauso können die Lernenden Rückmeldungen zum bisherigen Verlauf der Lehrveranstaltung geben. (vgl. z.B. Pelton / Pelton 2003 und Wessner et al. 2003).

Abb. 8. Multiple-choice Abfrage zur Überprüfung präsentierter Lerninhalte via PDA (Quelle: http://ipsi.fhg.de/concert/index_dt.shtml?projects_new/studeo)

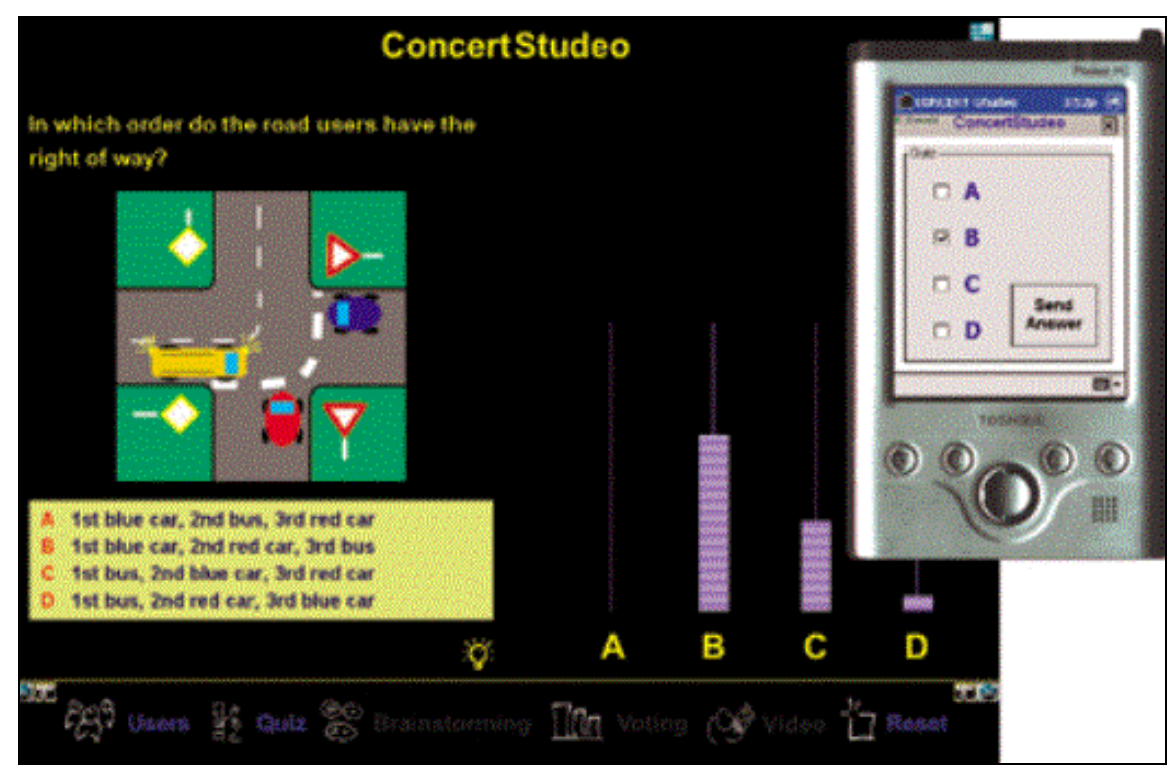

Großes Potenzial wird mobilen Endgeräten darüber hinaus dann bescheinigt, wenn es darum geht, informelle, kollaborative und ortsbezogene Lernaktivitäten zu ermöglichen (vgl. Oppermann / Specht 2003a und Schwabe 2003). Beispiele sind Exkursionen, in deren Verlauf Wasserproben analysiert oder Bilddokumentationen angefertigt werden können ${ }^{5}$, oder auch Schnitzeljagden für Erstsemester zur Orientierung in einer neuen Umgebung unter Nutzung mobiler Endgeräte (vgl. Abbildung 8).

5 Für Palm-PDAs sind Mess-Adapter und mehr als 25 verschiedene Sensoren (z.B. Temperatur, Licht, Geschwindigkeit, Gasanalyse, etc.) verfügbar (vgl. http://www.imagiworks.com/Pages/Products/ImagiWorksSensors.html). 
Abb. 9. Screenshots eines PDA-basierten Orientierungsspiels (Quelle: Göth 2003)

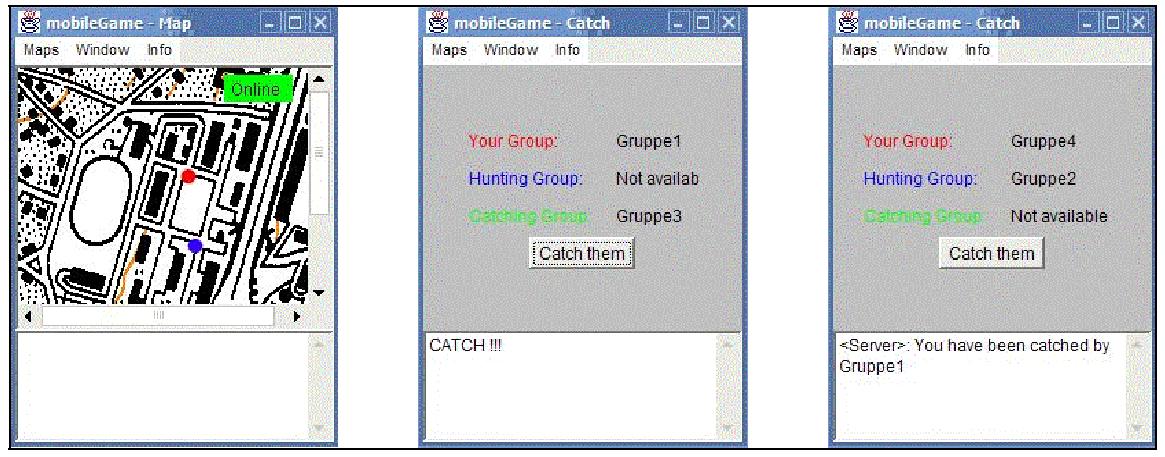

Mit der Verfügbarkeit von Mobilfunknetzen der dritten Generation (UMTS) und darauf aufbauenden ,location based services“ werden sich weitere neue Optionen ergeben. Denkbar sind etwa Informationsdienste wie die Benachrichtigung darüber, welche Mitglieder von studentischen Lern- und Arbeitsgruppen sich in der Nähe befinden.

So unterschiedlich die hier und im vorhergehenden Abschnitt aufgeführten didaktischer Szenarien sind, so unterschiedlich sind auch die neuen Möglichkeiten und der Mehrwert, der mit dem Einsatz mobiler Endgeräte und zu diesen kompatiblen Lerninhalten erzeugt werden kann: 
Tabelle 1. Lernformen und potenzieller Mehrwert von Szenarien mobilen Lernens

\begin{tabular}{|c|c|c|}
\hline Lernform & Beispiel & $\begin{array}{l}\text { Potenzieller Mehrwert } \\
\text { von Szenarien } \\
\text { mobilen Lernens }\end{array}$ \\
\hline $\begin{array}{l}\text { informelles, } \\
\text { non-curriculares } \\
\text { Lernen }\end{array}$ & Museumsbesuch & $\begin{array}{l}\text { Mobilität \& Flexibilität: } \\
\text { Ausstellungsdokumentation ist je- } \\
\text { derzeit zur Hand und kann u.U. spä- } \\
\text { ter weiter verwendet werden }\end{array}$ \\
\hline $\begin{array}{l}\text { individuelles } \\
\text { Lernen }\end{array}$ & Vokabellernen & $\begin{array}{l}\text { Mobilität: } \\
\text { Inhalte sind jederzeit zur Hand }\end{array}$ \\
\hline $\begin{array}{l}\text { kollaboratives } \\
\text { Lernen }\end{array}$ & $\begin{array}{l}\text { studentische } \\
\text { Arbeitsgruppe }\end{array}$ & $\begin{array}{l}\text { Mobilität \& Flexibilität: } \\
\text { Austausch von Dateien und Beteili- } \\
\text { gung an Diskussionsforen jederzeit } \\
\text { von jedem Ort }\end{array}$ \\
\hline $\begin{array}{l}\text { Lernen in } \\
\text { Kleingruppen }\end{array}$ & Seminar & $\begin{array}{l}\text { Flexibilität: } \\
\text { schneller Wechsel in Phasen der } \\
\text { Einzel- oder Gruppenarbeit (Web- } \\
\text { Recherche, Übungsaufgaben, etc.) }\end{array}$ \\
\hline $\begin{array}{l}\text { Lernen in } \\
\text { Großgruppen }\end{array}$ & Vorlesung & $\begin{array}{l}\text { Interaktivität: } \\
\text { bessere Aktivierung und Steuerung } \\
\text { durch Abfragen \& Rückmeldungen; }\end{array}$ \\
\hline $\begin{array}{l}\text { situiertes Lernen } \\
\text { im Transferfeld }\end{array}$ & $\begin{array}{l}\text { Exkursion; } \\
\text { Praktikum }\end{array}$ & $\begin{array}{l}\text { Intensität \& Authentizität: } \\
\text { Recherche \& Rezeption von Infor- } \\
\text { mationen im Feld praktischen Han- } \\
\text { delns; Messen \& Dokumentieren für } \\
\text { spätere Bearbeitung im Unterricht; }\end{array}$ \\
\hline
\end{tabular}

\subsection{Organisation}

Die Verbesserung der Lehre und die Flexibilisierung der Angebote durch Infrastrukturen und Inhalte für mobiles Lernen Umsetzung erfordert schließlich auch organisatorische Maßnahmen. Neben der Absicherung der Bereitschaft zur Veränderung unter den jeweiligen Stakeholdern (insbesondere Dozierende und IT-Abteilung) betrifft dies die Formulierung von Standards für die einzusetzenden Anwendungen und Inhalte, die schon oben (vgl. 3.2) angesprochene flächendeckende Versorgung mit mobilen Endgeräten und schließlich auch die Gewährleistung von IT-Sicherheit.

Im Bereich der in diesem Beitrag fokussierten mobilen Endgeräte wie PDAs und Smartphones besteht aktuell eine vergleichsweise große Heterogenität in Bezug auf Merkmale der Hardware (z.B. Displaygrößen), Be- 
triebssysteme, Anwendungen und nutzbare Datenformate (z.B. beim Media-Streaming). Damit stellt sich die Frage, wie Hochschulen mobil nutzbare Lernangebote bestmöglich verfügbar machen können. Ein möglicher Weg besteht darin, unterstützte Endgeräte-Klassen, Anwendungen und Datenformate klar zu definieren. Dies wird aber zwangsläufig dazu führen, dass nicht alle bereits bei den Studierenden verfügbaren Endgeräte genutzt werden können. Ein anderer Weg besteht darin, die mobilen Lernangebote konsequent auf standardisierte Datenformate wie html oder WML auszurichten, die dann mit unterschiedlichen Geräten genutzt werden können.

Weitere organisatorische Aufgaben im Zusammenhang mit mobilen Lernangeboten betreffen die Sicherung eines drahtlosen Netzwerks gegen unbefugte Zugriffe. Eine Option besteht darin, das flächendeckende Funknetz via Virtual Private Network (VPN) logisch vom eigentlichen CampusNetz zu trennen. Von den in das Funknetz eingebuchten Rechnern werden dann IPsec-Tunnel zu den Gateways aufgebaut, an denen eine zuverlässige Authentifikation erfolgt. Gleichzeitig kann die gesamte Nutzerkommunikation über das Funknetz nach WEP-Standard mit 128-bit Schlüsseln verschlüsselt werden (vgl. Schneider 2003, S. 189f.). Allerdings wird auch darauf hingewiesen, dass mit der Nutzung von Endgeräten wie PDA zum einen Sicherheitsrisiken durch den Verlust / Diebstahl von Geräten zunehmen und zum anderen die Umsetzung von Sicherheitsansprüchen mit den Anforderungen an eine unkomplizierte Nutzung dieser Endgeräte kollidieren (Forschungsgruppe Internet Governance 2003).

\section{Ausblick}

Die Verbreitung mobiler Endgeräte, die aufgrund ihrer geringen Größe immer zur Hand sein können, und die Einrichtung von Infrastrukturen für drahtlose Verbindungen in Netzwerke für Telefonie, Datenaustausch und das Internet verändern seit einigen Jahren unsere Kommunikationsgewohnheiten und eröffnen weiterhin neue Möglichkeiten für Arbeiten und Lernen. PDAs und Smartphones werden zu Schweizer Taschenmessern für das digitale Zeitalter. Sie erlauben mehr Flexibilität bei der Planung und Organisation von Lernaktivitäten und ermöglichen neue Szenarien des Lernens ebenso wie neue Services und Marketing-Strategien von Bildungsanbietern. Mobiles Lernen befindet sich zwar noch in einem sehr frühen, zumeist experimentellen Stadium. Dennoch handelt es sich hierbei um ein Thema, dass - ähnlich wie E-Learning - von strategischer Bedeutung für Hochschulen sein kann.

Einfache Lernaktivitäten, wie etwa das Repetieren von Lernstoffen mit digitalen Karteikarten-Systemen, werden zu jeder Zeit an jedem Ort mög- 
lich, an dem ein PDA oder Smartphone zur Hand ist. Auch etablierte Formen von Präsenz-Lehrveranstaltungen können mit neuen Elementen angereichert werden, etwa spontane und anonyme Abfragen unter den Teilnehmern zur Klärung, wie gut ein bislang behandelter Stoff bereits verstanden wurde. Neue Impulse gibt es auch für Formen des situierten Lernens, etwa im Rahmen von Exkursionen. Darüber hinaus können diese auf institutionalisierte Lernprozesse bezogenen Aktivitäten künftig unter Vermeidung von Medienbrüchen mit informellen Lernaktivitäten verknüpft werden: Die Hintergrund-Informationen, die man sich im Museum zu einer Ausstellung über die Künstlergruppe "Brücke" auf seinen PDA laden kann, kann man später auch für ein Referat zu diesem Thema im Rahmen des Studium Generale nutzen. Eine wichtige Voraussetzung für den erfolgreichen Einsatz von PDAs und Smartphones in Lernprozessen besteht allerdings darin, dass ein direkter Zugriff auf sehr kompakte Wissens- und Lerninhalte ohne umständliche Navigation möglich ist.

Mit diesen neuen Möglichkeiten und der Aussicht auf mehr Flexibilität sind aber auch Herausforderungen für Hochschulen (und natürlich auch andere Bildungsanbieter) verbunden. Wichtige Aspekte sind zum einen die Sorge dafür, dass mobile Endgeräte bei den Lernern flächendeckend verfügbar sind und dass hier keine Benachteiligungen für weniger vermögende Studierende entstehen. Zum anderen erfordert die flächendeckende Versorgung mit Zugängen zu universitären Netzwerken (W-LAN) ein hohes $\mathrm{Maß}$ an Aufmerksamkeit für die Sicherheit der Netzwerke.

\section{Literatur}

Bomsdorf, Birgit; Die virtuelle Universität macht mobil. In: Gesellschaft der Freunde der FernUniversität e.V. (Hrsg.), Jahrbuch 2003.

Bullinger, Hans-Jörg; Enterprise E-Learning - arbeitsprozessintegriertes Lernen. In: Dokumentation des Forums „E-Learning in Geschäftsprozessen. Arbeitsprozessbezogenes Lernen mit neuen Technologien“, hrsg. von H.-J. Bullinger \& O. Lindhorst. Stuttgart, Fraunhofer-IAO, 23. April 2002.

Forschungsgruppe Internet Governance, TU Berlin; Mobiler Zugang zu gesicherten Netzen. Evaluationsbericht für das BMI, 2003. (http://ig.cs.tuberlin.de/forschung/Mobile; 02.11.2003).

Göth, Christoph; Prototypische Implementierung einer mobilen Spielumgebung für den PDA. Diplomarbeit an der Universität Koblenz-Landau, 2003.

Jung, Volker \& Warnecke, Hans-Jürgen (Hrsg.); Handbuch für die Telekommunikation. 2., überarbeitete Auflage. Berlin u.a.: Springer, 2002.

Keegan, Desmond; The future of learning: From eLearning to mLearning. 2002. (http://learning.ericsson.net/leonardo/book.html; 02.11.2003). 
Lehner, Franz; Nösekabel, Holger; Schäfer Klaus J.; Szenarien und Beispiele für mobiles Lernen. Forschungsbericht Nr. 67 des Lehrstuhls für Wirtschaftsinformatik III, Universität Regensburg, November 2003.

Meier, Christoph; Wahl, Sibylle; Lindhorst, Odette; Holzschuh, Gabriele; eQtv Rich Media basiertes Lernen für kleine und mittlere Unternehmen; In: ELearning - Einsatzkonzepte und Erfolgsfaktoren des Lernens mit interaktiven Medien. 2., überarbeitete und ergänzte Auflage, hrsg. von U. Dittler. München: Oldenbourg, S. 247-264.

Nölting, Kristin; Notebook University Rostock. Mobilität, Flexibilität und Spontaneität im Studium. Folienpräsentation. (http://www.nur.unirostock.de/sites/aktuelles/aktuelles.htm; 02.11.2003).

Nyiri, Kristof; Towards a philosophy of m-learning. Paper presented at IEEE Worskhop on Wireless and Mobile Technologies in Education, Vaxjö University, Sweden, August 2002.

Oppermann, Reinhard; Specht, Marcus (2003a); Lernen im Betrieb mit MLearning gestalten. In: Handbuch E-Learning. Expertenwissen aus Wissenschaft und Praxis, hrsg. von A. Hohenstein \& K. Wilbers. Köln: Fachverlag Deutscher Wirtschaftsdienst, 5. Ergänzungslieferung August 2003.

Oppermann, Reinhard; Specht, Marcus (2003b); Mobiles Lernen. Erfahrungen und Perspektiven aus dem RAFT-Projekt. Vortrag im Rahmen der Session „Mobiles Lernen“ auf der Mensch\&Computer 2003, 09. September 2003, Stuttgart.

Pelton, Timothy W.; Pelton, Leslee Francis; The classroom interaction system (CIS): Neo-Slates in the classroom. In: Connections'03, ed. by W.-M. Roth, 2003, pp. 101-110. (http://www.educ.uvic.ca/Research/conferences/ connections2003/06PeltonT102.pdf ; 26.10.2003).

Schneider, Gerhard; Modernes Netzwerkmanagement: Grundlage für Multimedia und Mobilität in der Ausbildung. In: Wirkungen und Wirksamkeit Neuer Medien in der Bildung, hrsg. von R. Keil-Slawik \& M. Kerres. Münster u.a.: Waxmann, 2003, S. 185-194.

Schwabe, Gerhard; Mobiles Lernen: Evolution oder Revolution? Vortrag im Rahmen der Session „Mobiles Lernen“ auf der Mensch\&Computer 2003, 09. September 2003, Stuttgart.

Trondsen, Eilif; Mobile learning: A perspective on the present and the future. SRI Consulting Business Intelligence, LoD Bulletin First Quarter 2003.

Vahey, Phil \& Crawford, Valerie; Palm (TM) Education Pioneers Program: Final Evaluation Report. Palm, Inc., 2002. http://ctl.sri.com/publications/displayPublication.jsp?ID=115; 02.11.2003).

Wessner, Martin; Dawabi, Peter; Fernández, Alejandro; Supporting Face-To-Face Learning With Handheld Devices. In: In B. Wasson, S. Ludvigsen, \& U. Hoppe (Ed.): Designing for Change in Networked Learning Environments, Proceedings of the International Conference on Computer Support for Collaborative Learning 2003., pp. 487-491, Dordrecht, Kluwer, 2003. 